\title{
The effect of false-information feedback upon psychophysical judgments
}

\author{
Ronald A. Kinchla, AMES RESEARCH CENTER' \\ Hichard C. Atkinson, STANFORD UNIVERSITY
}

\begin{abstract}
Abstraet
An analysis was made of the effect of introducing false-information feedback in a yes-no signal detection task. The experimental results indicate that the falseinformation feedback increases both the probability of a hit and of a false alarm, and at the same time reduces the overall probability of a correct response.
\end{abstract}

\section{Introduction}

The present study was designed to assess the effect of introducing false-information feedback in a yes-no auditory detection task. The false information consisted of occasionally telling $S$ that a signal had occurred when in fact no signal was presented. Several detection theories represent $\mathrm{S}$ as adjusting his decision process or response bias in accord with the proportion of trials on which a signal is presented; the higher the proportion of signal trials the more frequently $\mathrm{S}$ will report a signal, both on signal and on no-signal trials (e.g., Atkinson, 1963; Luce, 1963; Swets et al., 1961). The typical method for providing information feedback in a detection task is to tell $\mathrm{S}$ whether his response was correct or incorrect at the end of each trial. Since the stimulus variable (signal or no-signal) is perfectly correlated with the feedback variable, it is not possible to partial out the effect of each variable in determining the S's response bias. Introducing false-information feedback is one way of assessing the relative influence of these two variables. The proportion of trials on which a subject is told a signal has occurred can be manipulated independently of the actual signal presentation schedule. However, if $\mathrm{S}$ were to clearly detect a signal and were then told that none had been presented, he might doubt the accuracy of the feedback. In order to eliminate this possibility, in the present study false information was given only on no-signal trials; i.e., a $\mathrm{S}$ was never told that a signal did not occur on a signal trial.

On each detection trial an observation interval was defined for $\mathrm{S}$; he then indicated whether or not he thought a signal (tone burst) had been added to a constant background of white noise during that interval. Each trial was terminated with a feedback event which ostensibly indicated whether or not a signal had been presented. A trial on which a signal occurred will be denoted $S_{1}$, and a trial on which no signal occurred will be denoted $S_{2}$. The S's response will be denoted as $A_{1}$ if he reported a signal, and $\mathrm{A}_{2}$ if he reported no signal. Feedback indicating the supposed occurrence of signal or no-signal will be denoted as $E_{1}$ or $E_{2}$, respectively. In the present study three types of trials were used: $\mathrm{S}_{1} \mathrm{E}_{1}, \mathrm{~S}_{2} \mathrm{E}_{1}$, and $\mathrm{S}_{2} \mathrm{E}_{2}$. The first indicates a trial on which a signal occurred and $\mathrm{S}$ was correctly informed that a signal had been presented; the second indicates a trial on which no signal was presented and $S$ was falsely told that a signal had occurred; the third indicates a trial on which no signal occurred and $\mathrm{S}$ was correctly informed that no signal had been presented. We denote the probabilities of these trial types as follows: $\operatorname{Pr}\left(\mathrm{S}_{1} \mathrm{E}_{1}\right)=\gamma, \operatorname{Pr}\left(\mathrm{S}_{2} \mathrm{E}_{1}\right)=(1-\gamma) \lambda$, and $\operatorname{Pr}\left(\mathrm{S}_{2} \mathrm{E}_{2}\right)=(1-\gamma)$ $(1-\lambda)$. Thus, $\gamma$ is the probability of presenting a signal trial, and $\lambda$ is the probability of giving false-information feedback on a no-signal trial. The following four experimental conditions were run in this study: for the first condition $\gamma=.25, \lambda=0$; for the second $\gamma=.25, \lambda=.33$; for the third $\gamma=.50, \lambda=0$; and for the fourth condition $\gamma=.50, \lambda=.50$. Thus, over the four experimental conditions, the probability of a signal took on the values .25 , $.25, .50$ and .50 ; whereas, the corresponding probability of an $\mathrm{E}_{1}$ event was $.25, .50, .50$ and .75 .

\section{Method}

Gaussian noise was presented binaurally in each S's headphones (Permoflux PDR-8) throughout a test session. The signal was a $1000 \mathrm{cps}$ sinusoid presented for $100 \mathrm{msec}$, including $10 \mathrm{msec}$. rise and decay times. The ratio of signal energy to noise power in a one cycle band was a constant 2.9 throughout the study. Each $\mathrm{S}$ was seated before a display-response panel in a separate booth (Industrial Acoustics 400-A). A blue warning light came on for $100 \mathrm{msec}$. to indicate the beginning of each trial. Then, after a delay of $500 \mathrm{msec}$, a red light came on for $100 \mathrm{msec}$. and defined the observation interval. The $\mathrm{S}$ had $2.5 \mathrm{sec}$. following the observation interval to make his response by pressing either a button labeled "signal" or one labeled "no signal." The trial then ended with the illumination of one of these two buttons for $700 \mathrm{msec}$. to indicate the "correct" response for that trial. A $1.1 \mathrm{sec}$. intertrial period was used; hence each trial lasted for $5 \mathrm{sec}$.

The Ss were 14 college students with normal hearing. They were run for six practice sessions followed by 24 test sessions. Test sessions were run on consecutive days, 400 trials/day. On each test day $S$ ran on 1 of the 4 experimental conditions; in successive 4-day blocks $\mathrm{S}$ ran 1 day on each of the 4 experimental conditions in a random order. Thus, over 24 test days each of the experimental conditions was repeated 6 times.

\section{Results and Discussion}

Inspection of these data indicates that response measures over trials 101-400 for the last four test days on each experimental condition are quite stable, and may be regarded as representing asymptotic performance. All 
PLOTS OF $\operatorname{Pr}\left(A_{1} \mid S_{1}\right)$ AGAINST $\operatorname{Pr}\left(A_{1} \mid S_{2}\right)$ FOR INDIVIDUAL SUBJECTS AND THE GROUP AVERAGE

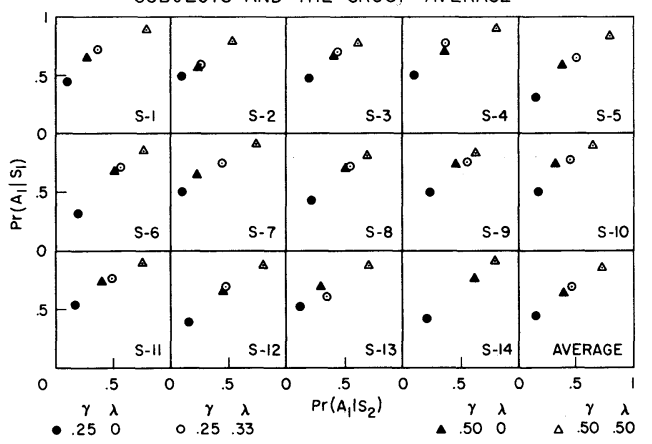

Fig. 1.

results presented here refer to that part of the data.

Table 1 gives the proportion of $\mathrm{A}_{1}$ responses on both $S_{1}$ trials (hits) and on $S_{2}$ trials (false alarms), for each of the four experimental conditions. These same statistics are plotted in Fig. 1 as points in the familiar response-operating-characteristic (ROC) space. The two points obtained for the experimental conditions where $\lambda=0$ represent data from a typical detection task; for most signal-detection theories these two points suffice to define an ROC curve. An important theoretical question is whether the introduction of false information on no-signal trials gives rise to points that fall on the ROC curve defined by the experimental conditions involving no misinformation. Some theories require that all four points should fall on the same ROC curve, whereas others predict that the points associated with the misinformation conditions should fall below the ROC curve defined by experimental conditions involving no false information. The reader may inspect the individual ROC plots to decide which conjecture is the more reasonable; it is our opinion that these data favor the hypothesis that, for a given subject, the four points fall on the same ROC curve. In fact, a simple linear ROC curve would provide a reasonably good account of almost every S's data.

The ordering of the points in the ROC space is extremely consistent. For every $\mathrm{S}$, the $(\gamma=.25, \lambda=0)-$ condition generates an extreme point in the lower-left area of the ROC space, whereas the $(\gamma=.50, \lambda=.50)-$ condition generates an extreme point in the upper-right area. Further, for 13 of the 14 S's the point associated with the $(y=.25, \lambda=.33)$-condition is to the upper right of the point for the $(\gamma=.50, \lambda=0)$-condition. This last comparison is particularly interesting, because the overall probability of an $E_{1}$ event is .50 for both of
TABLE I

$\operatorname{Pr}\left(A_{1} I S_{1}\right), \operatorname{Pr}\left(A_{1} I S_{2}\right), \operatorname{Pr}(C)$, AND $\operatorname{Pr}\left(A_{1}\right)$ UNDER THE FOUR EXPERIMENTAL CONDITIONS FOR INDIVIDUAL SUBJECTS AND AN OVERALL AVERAGE

\begin{tabular}{|c|c|c|c|c|c|c|c|c|c|c|c|c|c|c|c|c|}
\hline \multirow{2}{*}{ SUBBECT } & \multicolumn{4}{|c|}{$\boldsymbol{y}=25, \lambda=0$} & \multicolumn{4}{|c|}{$\boldsymbol{\gamma}=.25, \lambda=.33$} & \multicolumn{4}{|c|}{$\gamma=.50, \lambda=0$} & \multicolumn{4}{|c|}{$\gamma=.50, \lambda=50$} \\
\hline & $A_{1}\left(S_{2}\right)$ & Pr $\left(\mathrm{A}, \mathrm{I}_{2}\right)$ & $P r(C)$ & $\operatorname{Pr}\left(A_{1}\right)$ & Pr(A,,$\left.S_{S}\right)$ & $\operatorname{Pr}\left(A, S_{2}\right)$ & Pritc) & $P r\left(A_{1}\right)$ & Pri $, I_{1},{ }_{1}$ & $\operatorname{Pr}_{r}\left(A_{1}, S_{2}\right)$ & PriC) & $\operatorname{Pr}\left(\mathrm{A}_{1}\right)$ & PriA, $\left.I_{2}\right)$ & (1) $P\left(A_{1}, S_{2}\right)$ & PrC) & $\operatorname{Pr}\left(A_{1}\right)$ \\
\hline 1 & .452 & .10 & .787 & .189 & & .360 & .663 & .454 & 60 & & 38 & .462 & .905 & & 359 & 46 \\
\hline 2 & & .0 & .807 & .196 & & & .709 & .343 & & & .669 & .403 & & .528 & 33 & 61 \\
\hline 3 & & .19 & .728 & .267 & & & .5 & .511 & & & .632 & .540 & 80 & .611 & .585 & 695 \\
\hline 4 & & & .801 & .20 & & & .667 & & & & .675 & .536 & 112 & 11 & 551 & \\
\hline 5 & & .1 & .726 & & & & .552 & & & & 615 & .479 & & .779 & 531 & 31 \\
\hline 6 & & .19 & $.69 \mathrm{C}$ & .22 & & & .51 & .599 & & 1 & .590 & .591 & 860 & .759 & .551 & .809 \\
\hline 7 & & .104 & .80 & .20 & & .44 & .60 & .524 & & & .712 & .439 & 15 & .737 & .589 & 826 \\
\hline 8 & & .22 & .69 & .27 & & & .51 & .60 & & & 993 & .614 & 77 & & & \\
\hline 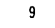 & & & .7 & & & & & .59 & & & .650 & .593 & 48 & .615 & .617 & 731 \\
\hline 10 & & .160 & .75 & .246 & .78 & & .61 & .524 & & .306 & .720 & .526 & 103 & .635 & .634 & .769 \\
\hline 11 & & .16 & .763 & .26 & .7 & .48 & .577 & .555 & & & .671 & .571 & 31 & 756 & 568 & 323 \\
\hline 12 & .4 & .155 & .734 & .217 & 6 & .4 & .566 & .531 & & & & .555 & & & & 340 \\
\hline 1 & & .1 & .79 & & & & & & & & .6 & 499 & & .718 & .581 & .799 \\
\hline 14 & & .205 & .701 & .258 & .777 & .615 & .482 & .655 & $.1 / 4$ & .615 & .580 & .695 & .914 & .791 & .562 & .853 \\
\hline$R$ & & .155 & .749 & .232 & .722 & .457 & .588 & .523 & .686 & .386 & .650 & .536 & .868 & .716 & .576 & .792 \\
\hline
\end{tabular}

these conditions. Although $\mathrm{S}$ is told that the signal and no-signal trials are occurring equally often in these two conditions, nevertheless, he tends to have a stronger $\mathrm{A}_{1}$ bias for the $(\gamma=.25, \lambda=.33)$-schedule. Thus, while it is clear that feedback plays an important role in determining the response bias, there is also an interactive effect with the stimulus presentation schedule. It should be noted that the observed arrangement of points in the ROC space are well accounted for by the model proposed by Atkinson (1963).

Table 1 also presents the overall probability of a correct response and of an $\mathrm{A}_{1}$ response; i.e.,

$$
\begin{aligned}
\operatorname{Pr}(\text { correct }) & =\gamma \operatorname{Pr}\left(\mathrm{A}_{1} \mid \mathrm{S}_{1}\right)+(1-\gamma) \operatorname{Pr}\left(\mathrm{A}_{2} \mid \mathrm{S}_{2}\right) \\
\operatorname{Pr}\left(\mathrm{A}_{1}\right) & =\gamma \operatorname{Pr}\left(\mathrm{A}_{1} \mid \mathrm{S}_{1}\right)+(1-\gamma) \operatorname{Pr}\left(\mathrm{A}_{1} \mid \mathrm{S}_{2}\right)
\end{aligned}
$$

The introduction of false-information feedback both increases the probability of an $\mathrm{A}_{1}$ response and decreases the probability of a correct response. By inspection of the table one can see that these relations hold for every S. It should be emphasized that these effects can be explained in terms of a simple response-bias process (Atkinson, 1963), and do not necessitate assuming that S's sensitivity level is lower under conditions of misinformation feedback.

\section{References}

A TKINSON, R. C. A variable sensitivity theory of signal detection. Psychol. Rev., 1963, 70, 91-106.

LUCE, R. D. A threshold theory for simple detection experiments. Psychol. Rev., 1963, 70, 61-79.

SWETS, J. A., TANNER, W. P., JR., \& BIRDSALL, T. G. Decision processes in perception. Psychol. Rev., 1961, $68,301-340$.

\section{Note}

1. Now at New York University. 